\title{
Linx
}

Revue des linguistes de l'université Paris X Nanterre

47 | 2002

Du sens au sens

\section{Les titres de Magritte : surprise et convenance discursive}

Magritte's titles : surprise and discursive adequacy

\section{Bernard Bosredon}

\section{(2) OpenEdition}

\section{Journals}

Édition électronique

URL : http://journals.openedition.org/linx/577

DOI : $10.4000 /$ linx.577

ISSN : 2118-9692

\section{Éditeur}

Presses universitaires de Paris Nanterre

\section{Édition imprimée}

Date de publication : 1 décembre 2002

Pagination : 43-54

ISSN : 0246-8743

\section{Référence électronique}

Bernard Bosredon, «Les titres de Magritte : surprise et convenance discursive », Linx [En ligne],

47 | 2002, mis en ligne le 01 juin 2003, consulté le 19 avril 2019. URL : http://journals.openedition.org/ linx/577; DOI : 10.4000/linx.577 


\section{Les titres de Magritte : surprise et convenance discursive}

Bernard Bosredon Paris 3 - Sorbonne Nouvelle

«La poésie est une pipe » René Magritte, 1929.

\section{Introduction $^{1}$}

René Magritte est certainement l'un des peintres qui intriguent le plus les philosophes, les logiciens ou les linguistes tant sa peinture entretient avec les mots un commerce intime et étrange à la fois, qu'il en fasse un motif de la toile ou plus conventionnellement son titre. La même curiosité en effet le pousse vers les mots et les images de sorte qu'il les pense toujours ensemble, dans des relations qui les concernent également ou qui en soulignent le contraste. Relations de dénomination («Un objet ne tient pas tellement à son nom qu'on ne puisse lui en trouver un autre qui lui convienne mieux»), relation de substitution («Une image peut prendre la place d'un mot dans une proposition»), relations sémantiques présentées de façon contrastive («On voit autrement les images et les mots dans un tableau » ou encore «Les mots qui servent à désigner deux objets différents ne montrent pas ce qui peut séparer ces objets l'un de l'autre ») ou opposées ensemble et parallèlement au plan du référent («Un objet ne fait jamais le même office que son nom et son image ») ${ }^{2} .$. On pourrait allonger considérablement cette liste de citations. Elles jalonnent en effet tous les textes de Magritte. Désireux de témoigner de l'intérêt que portait Michel Galmiche aux liens possibles entre les images peintes et les mots (ou les textes) qui leur sont associés dans le cartel du titre, parfois dans la dépiction même, je voudrais retrouver, l'espace de quelques pages, l'écho d'interrogations qu'il acceptait d'entendre ou

${ }^{1}$ Qu'il me soit permis de remercier ici Rémy Porquier pour l'apport précieux de ses remarques. La perspective toute personnelle adoptée ici explique l'absence de références aux études classiques et spécialisées concernant le surréalisme. J'ajouterai enfin que la bibliographie est réduite aux seuls ouvrages exploités.

${ }^{2}$ Cf. Écrits complets, p. 60. 


\section{Bernard Bosredon}

d'émettre lui-même, toujours de bonne grâce, avec sa compétence de linguiste et son talent de peintre.

La première question à laquelle on est immédiatement confronté, c'est d'abord l'apparent parti pris chez Magritte d'un décalage entre les images et les mots. Nous commencerons donc par cette énigme et nous verrons comment la non-adéquation patente entre la peinture et le langage est certainement l'effet de constructions sciemment entreprises où le hasard tient une place relativement réduite, voire inexistante. Magritte ne s'en est pas caché. Il explique longuement dans les Écrits complets ainsi que dans les Lettres à André Bosmans que l'emploi très personnel qu'il avait des mots dans sa peinture avait pour origine une alchimie, élaborée souvent collectivement, et qui tendait à une poétique qu'on qualifiera de «verbo-picturale », les mots et les images peintes concourant ensemble selon lui à la création artistique.

La deuxième énigme offre l'aspect paradoxal et inattendu d'une intitulation à certains égards sans surprise. Autant Magritte s'est en effet longuement interrogé sur le rapport entre les mots et la peinture, autant il a été, à ma connaissance, silencieux sur le choix de la forme linguistique dans laquelle ce rapport pouvait s'exprimer. Cela se traduit par l'existence d'une structure discursive visiblement classique, à la stabilité jamais vraiment menacée par l'absence d'adéquation entre ce que la dépiction montre et ce que le titre stipule ou encore par la présence, ici ou là, de ces quelques titres isolés, atypiques et marginaux, construits sur le schéma bien connu de CECI EST/N'EST PAS UN $\mathrm{X}^{3}$. Ce manque d'intérêt pour la forme du titre correspond-il à une décision mûrie ? et consciemment exprimée par un titreur auteur? Magritte ne semble pas avoir revendiqué cette fidélité à l'usage. Il appliquerait donc un formulaire plus qu'il ne le construirait et se situerait, de ce point de vue, et peut-être à son corps défendant, dans la tradition qu'il contribue pourtant à dénoncer.

Nous voudrions montrer ainsi qu'une peinture révolutionnaire peut s'accommoder d'une intitulation sage et conforme aux usages établis, et suggérer par là que les jeux de langage voulus par un sujet parlant ne touchent pas nécessairement aux bases d'un processus discursif global comme celui de l'intitulation picturale. Pour cela, nous commencerons par montrer l'aspect transgressif de l'intitulation magrittienne, sa dimension de rupture avec l'intitulation classique, pour rappeler ensuite les grands principes sur lesquels se fonde cette nouvelle poétique du titre de peinture. En nous appuyant sur quelques indices formels et sur le statut sémiotique des titres, nous verrons enfin comment l'invention des titres reste chez Magritte dans les limites d'une discursivité relativement stable.

\footnotetext{
${ }^{3}$ Comme Ceci est un morceau de fromage (1936-1937), Ceci n'est pas une pomme (1964) et bien sûr les deux Ceci n'est pas une pipe, "surnom » donné à la fois à La trabison des imges de 1928-1929 et à la toile de 1966 Les deux mystères. Pour être complet, il convient d'ajouter à ces titres non standard du corpus magrittien le titre Pom'po pom'po pom po pom pon, une gouache de 1947/48 figurant un lapin jouant du tambour...
} 


\section{Rupture apparente de l'intitulation magrittienne avec l'intitulation classique}

Magritte n'est pas le premier à avoir transgressé les lois implicites de l'intitulation classique. Voilà la femme ! de Picabia ou plus encore le titre L.H.O.O.Q. du même peintre, réintitulant une reproduction de La Joconde, ont défrayé la chronique en leur temps. Plus discrètement, Femmes qui courent de Picasso apporte une note spécifique, la relative étant d'ordinaire peu usitée dans les titres de tableaux ${ }^{4}$. S'il n'est certes pas le premier à avoir fait bouger le système de l'intitulation classique, Magritte a joué un rôle important dans l'histoire de cette pratique en faisant porter principalement sa critique sur deux de ses principes fondateurs: le principe d'extériorité qui fait du titre l'interprétant linguistique d'un élément non linguistique, l'interaction titre/peinture conçue traditionnellement comme une légende explicative.

\subsection{Critique du principe d'extériorité}

Comme des légendes dans un dispositif d'étiquetage, les séquences verbales ayant un rôle de titre sont dans une position d'extériorité par rapport à la toile. Or chez Magritte les mots ne se concentrent pas uniquement dans la zone réservée aux titres. Il peuvent aussi apparaittre dans la peinture elle-même. Nombreuses en effet sont les peintures qui comportent à la fois des figurations de choses et des figurations de mots. L'espoir rapide de 1927 ou Le miroir vivant de 1928/29 donnent à voir des formes vagues (taches ou nuages plus ou moins en contraste avec un fond monocolore) auxquelles sont associés par contiguité des mots ou des syntagmes comme arbre, nuage, village à l'horizon, cheval, chaussée de plomb pour la première toile, personnage éclatant de rire, horizon, cris d'oiseau, armoire pour la deuxième. Des mots écrits par conséquent en lieu et place de choses peintes.

Le peintre déplace ainsi les frontières qui séparent les mots et la peinture dans deux zones distinctes et hétérogènes ou rendent poreux ce qui devrait les séparer. Cela s'observe dans un chassé-croisé intéressant entre les mots sur la toile et ce qui en fait son titre. On voit ainsi une huile de 1926 représentant une pipe grossièrement reproduite porter la désignation de l'objet (la pipe). Cette peinture dont le titre est précisément de n'en avoir aucun (Sans titre) peut cependant être identifiée par ajout de cette désignation interne à la peinture (la pipe), dans une sorte de transfert métonymique. On peut lire ainsi dans le René Magritte de Jacques Meuris (Meuris, 1990) ce titre composite Sans titre (la pipe) qui intègre à la fois l'élément verbal interne (la pipe) et le titre primitif (Sans titre). La répartition des mots ressortissant du titre d'une part, des mots figurant dans la peinture d'autre part, n'est donc pas une répartition imperméable, les mots de la toile étant ici promus au rang d'un titre accessoire. On pourra observer le même transfert avec La trabison des images de 1928/29 et Les deux mystères de 1966, chaque œuvre étant métonymiquement réintitulée au moyen du fameux Ceci n'est pas une pipe peint dans chacune des toiles.

${ }^{4}$ B. Bosredon, 1997, p. 87. 
Nous retiendrons donc que le dispositif d'étiquetage qui corrèle un titre légende et son objet n'est plus avec Magritte le seul système d'assemblage des mots et de la peinture. Il préfère une formule qui les associe dans un même espace ${ }^{5}$ comme l'attestent ces deux phrases de La ligne de vie $I^{6}$ : « Dans un tableau, les mots sont de la même substance que les images » et «Une image peut prendre la place d'un mot dans une proposition». Étant donné toutefois les limites de cette étude, nous nous concentrerons sur ce qui apparait principalement dans la zone du titre, en gardant néanmoins en mémoire que les mots chez Magritte peuvent déborder les limites d'un simple cartel sous une toile ou celles d'une légende dans un catalogue.

\subsection{Critique de la fonction justificative du titre}

Nous avons vu plus haut que les titres classiques présentent une sorte d'autonomie par rapport aux toiles, cette autonomie étant sémiotiquement attestée par une répartition traditionnelle en deux zones distinctes du langage et de l'image, le cartel du titre d'une part, le cadre de la toile d'autre part. Cependant, les titres restent toujours dans une relation de dépendance par rapport aux tableaux car s'il existe des peintures sans titre, on ne peut pas concevoir de titres sans peintures. L'existence d'un titre présuppose donc celle d'une toile dont il est comme sa contrepartie dans un texte, un catalogue, etc. Les titres sont donc aux toiles ce qu'un reçu est à un achat: un justificatif ; justificatif d'achat dans un cas, justificatif d'existence dans l'autre. Dans Un cabinet d'amateur, Georges Pérec relate l'histoire d'un tableau. Le vrai et le faux se mêlent certainement tout au long du récit mais toute mention de titre implique et atteste l'existence réelle ou fictionnelle d'une toile ${ }^{7}$.

Or cette fonction justificative du titre qui évoque de façon parallèle la présupposition d'existence impliquée par l'activité de nomination fait davantage encore. En tant que légendes, les titres construisent aussi une représentation qui entre nécessairement en rapport avec ce que produit la peinture chez l'observateur. L'intitulation classique est conçue comme un apport assurant non seulement un complément verbal à ce que l'observateur voit et comprend mais également comme une façon de fixer, dans les limites généralement d'une désignation et grâce à elle, ce qu'une image ne peut à soi seule déterminer tant son interprétation est inépuisable. Un titre arrête donc le mouvement interprétatif comme des marges bordent un texte en le « justifiant» précisément. Titre-justificatif donc en ce qu'il stipule et légitime, dans la co-présence de la toile, ce que la peinture est censée produire dans la tête de celui qui l'examine.

Magritte rompt avec cette conception du titre: «Les titres de tableaux ne sont pas des explications et les tableaux ne sont pas des illustrations des titres $»^{8}$. Il dénonce cet ajout verbal a minima qu'est le titre classique, senti comme un simple doublon

\footnotetext{
${ }^{5}$ Magritte pense que mots et images sont deux modes d'une seule et même réalité. L'art de donner à voir cette dernière est mieux servie si l'on peut se servir simultanément des deux modes.

6 in Ecrits complets, p. 121.

${ }^{7}$ Cf Mathieu Lindon ; quatrième de couverture de l'édition du Livre de Poche de Un cabinet d'amateur (Pérec, 1999).

${ }^{8}$ Écrits complets, p. 259.
} 
linguistique d'une figuration tout aussi transparente. Ainsi la représentation d'une laitière ou d'un joueur de fifre sera-t-elle intitulée La laitière et Le joueur de fifre - sans vraie surprise. Dans un tel dispositif où le verbal répète en quelque sorte l'image, les titres n'entretiennent pas d'interaction véritable avec la figuration, sinon sur le mode de la désignation et/ou du commentaire explicatif tel qu'on le voit à l'œuvre dans la légende sous image. Il préfère aux situations d'accord entre ce que le titre exprime et ce que la peinture donne à voir les situations dans lesquelles le rapport entre les titres et les peintures pose problème et présente une énigme au lecteur-observateur de l'ensemble titre-toile.

\section{Construire l'énigme : vers une nouvelle poétique du titre de peinture}

Les images, les mots et les idées ne constituent pas pour Magritte trois ordres séparés mais trois modes spécifiques de la même réalité : la pensée 9 . Il s'en suit que les mots peuvent être reçus comme des images et les images comme des mots. La non séparabilité des trois modes d'accès à la vraie réalité permet d'établir un parallèle entre les images d'objets et les dénominations d'objets ou, plus exactement, les dénominations d'images d'objets. Tout en croyant profondément à l'unité de cette réalité qu'il se propose de révéler, Magritte n'a de cesse de montrer le caractère illusoire de l'expérience ordinaire du langage et des images en dérangeant nos habitudes perceptives.

\subsection{Déranger nos perceptions}

On peut donc dire que, de ce point de vue, l'artiste, selon Magritte, est comme un pédagogue. Il fabrique en effet d'ingénieux dispositifs images/textes pour donner à voir, à sentir et à penser de la nouveauté, la leçon du jour. Pour cela, il doit piquer la curiosité. Les déplacements dont nous avons parlé sont autant de moyens d'alimenter cette curiosité. Ainsi, en effaçant les frontières spontanément reconnues par un lecteur/observateur entre les mots et les images, ces frontières qui délimitent, avec la répartition cartel/cadre, des zones sémiotiques hétérogènes d'une part, et ces autres frontières qui contiennent des représentations de nature sémantique bien claires d'autre part, Magritte se sert des mots pour doter ses images d'un pouvoir énigmatique. L'énigme n'est pas rencontrée par le peintre, elle est le fruit d'un travail. Elle est construite.

Comme l'écrit en effet Paul Nougé ${ }^{10}$ dans Les images défendues, l'énigme a pour origine une reconstruction de la réalité à partir de la déconstruction de la logique spontanée, ce qui va évidemment dans le droit fil de l'esprit surréaliste. Les deux premiers procédés portent sur le référent, c'est-à-dire sur la dépiction. Magritte commence en effet par « dépayser » les objets. Il faut pour cela que l'objet «à traiter» soit le plus familier possible. Soit par exemple d'un côté un enfant, de l'autre une

9 "...les images, idées et paroles sont des déterminations différentes d'une seule chose : la pensée », Écrits complets, p. 379.

${ }^{10}$ Les images défendues, cité par Magritte dans Écrits complets, p. 110. 


\section{Bernard Bosredon}

planète en feu ; «un enfant en feu nous touchera davantage qu'une planète lointaine se consumant » écrit Magritte. Le deuxième procédé consiste à transformer la matière du référent, à assimiler au contraire ce qui ne peut être confondu ou à inciter le spectateur à percevoir des objets nouveaux ou des situations nouvelles. Magie par conséquent de la peinture avec ses hommes et ses lions de pierre ou bien encore ses images qui ont la forme de mots ou qui sont faites de mots comme L'art de la conversation par exemple ${ }^{11}$. L'appel des cimes ou La condition humaine assimilent l'objet peint (un paysage) et sa reproduction, ravalant la toile à un simple vitrage. La recherche du peintre consiste donc à faire coexister sur une toile ce qui ne coexiste pas hors d'elle. Au bout du processus, le mystère: «Les objets ne se présentent pas comme mystérieux, c'est leur rencontre qui produit du mystère $»^{12}$.

\subsection{Le titre-énigme}

Le troisième procédé du «dépaysement» concerne enfin les titres. Jusqu'ici « commodité de la conversation » selon Magritte, ils participent désormais activement à cette entreprise de dérangement généralisé. De même que les objets ne sont pas en soi mystérieux et que c'est la coprésence d'objets familiers qui peut les rendre tels, de même c'est la rencontre de mots non mystérieux et de figurations non mystérieuses qui produisent le mystère. Ils ne devront donc ni expliquer, ni rassurer: «les titres sont choisis de telle façon qu'ils empêchent aussi de situer mes tableaux dans une région rassurante que le déroulement automatique de la pensée lui trouverait afin de sous-estimer leur portée ${ }^{13}$. Déranger pour reconstruire, déconstruire pour déranger, tels sont les deux principes complémentaires de l'esthétique magritienne ouvrant la voie à une nouvelle poétique du titre. Dans ces conditions, l'invention du titre devient une expérience cruciale puisqu'il participe pleinement à la production esthétique, avec ce que cela implique de sérieux et de nécessaire: "Les titres doivent être une protection supplémentaire qui découragera toute tentative de réduire la poésie véritable à un jeu sans conséquence $»^{14}$.

Cette recherche du bon titre ressemble à une forme d'obsession. La correspondance avec André Bosmans est émaillée de demandes, de suggestions, de propositions fermes souvent. On raconte que, réunis autour du tableau, Magritte et ses amis avançaient des titres dont un seul était finalement retenu pour sa seule valeur énigmatique. Parfois le mot ne dénomme rien. C'est le cas du Prisonnier, toile

\footnotetext{
11 Magritte détaille ainsi les différents procédés de cette technique du dépaysement : «La création d'objets nouveaux; la transformation d'objets connus; le changement de matière pour certains objets ; un ciel de bois, par exemple ; l'emploi de mots associés aux images ; la fausse dénomination d'un objet; la mise en oeuvre d'idées données par des amis ; la représentation de certaines visions du demi-sommeil ; furent en gros les moyens d'obliger les objets à devenir enfin sensationnels et à établir un contact profond entre la conscience et le monde extérieur ", Écrits complets, p. 120.

12 Ecrits complets, p. 371. ; ibid. p. 378 : «L'image d'une locomotive est immédiatement familière, son mystère n'est pas perçu. Pour que son mystère soit évoqué, une autre image immédiatement familière - sans mystère - l'image d'une salle à manger a été réunie à l'image de la locomotive... ».

${ }^{13}$ R. Magritte (Écrits complets, p. 110).

14 ibid.
} 
composée de formes vagues où l'on chercherait vainement l'ombre d'un personnage répondant à cette dénomination. Parfois, c'est une partie seulement du mot qui fait défaut comme dans ce titre Baron intitulant une toile figurant un «bas rond $»^{15}$, l'articulation toile/titre invalidant le mot écrit dans la promotion calembour d'une autre forme. Derrière un pseudo-titre, un autre titre donc. Nous verrons plus loin ce qu'il faut penser des titres dénégation du type Ceci n'est pas une pomme.

Il y a chez Magritte cette conviction solidement ancrée que le sentiment d'y voir clair en quelque manière est l'effet d'un désir traitre de clarté ${ }^{16}$. Tout nous pousse à constituer la plénitude du sens, sous quelque forme que ce soit, notamment dans l'articulation dans le titre des mots et des choses. Illusion de la liberté. Nous sommes en effet victimes de nos habitudes de pensée, de parler et d'imaginer ${ }^{17}$. C'est pourquoi faire en sorte que coexiste, dans les toiles et dans leur articulation aux titres, ce qui n'existe pas «à l'état naturel » est l'objet de cette recherche. Pour cela évidemment, l'artiste doit être convaincu de l'aspect problématique du banal: «Je ne puis douter que le monde soit une énigme $»^{18}$. S’il peut par conséquent constituer des dispositifs image/texte mystérieux, c'est que le monde lui-même est mystérieux. Il n'invente pas les problèmes, il se contente de montrer qu'ils existent ${ }^{19}$.

\section{Les limites de l'intitulation magritienne}

En s'interrogeant grâce à l'intitulation sur le caractère problématique des images et du langage, en montrant au moyen de dénominations décalées que les dénominations ad hoc constituent, dans l'inconscience des locuteurs, des bornes excessives si l'on veut donner tout leur essor aux forces de l'imaginaire, Magritte s'est lui-même pris à son tour au piège de déterminations inconscientes : les limites de la désignation d'une part, celle d'une forme discursive stéréotypée d'autre part.

\subsection{L'emprise de la désignation}

On constate en effet que, à l'exception des quelques titres de type CECI (N') EST (PAS) UN X, les titres sont dans leur grande majorité des $\mathrm{SN}$ courts composés d'un nom suivi d'un Adjectif (Le modèle rouge, 1937), d'un syntagme prépositionnel (Les épaves de l'ombre, 1926) voire simplement d'un nom précédé d'un article défini ( $L a$ fenêtre, 1925). Pas de phrase, pas de liste. Une concentration sur l'objet ou l'idée naturellement désignés par des SN simples. Ces formes sont des désignations

${ }^{15}$ Le vêtement féminin.

${ }^{16}$ La trahison des images exprime explicitement cette idée.

17 «L'habitude de parler pour les besoins immédiats de la vie impose aux mots qui désignent les objets un sens limité. Il semble que le langage courant fixe des bornes imaginaires à l'imagination » (Magritte, 1979, p.120).

18 R. Magritte (Lettres à André Bosmans, 1990, p. 153).

19 «Je ne puis douter que le monde soit une énigme (non pas une énigme à résoudre par quelque « discipline » que ce soit). Cette certitude ressemble à ce que le monde nous offre. Je dirais donc que les problèmes se posent à nous et non comme vous me l'écrivez: que nous pouvons poser les problèmes » (Magritte, 1990, p.153). 
nominales (ou des SN désignatifs comme l'on veut) au sens où elles désignent au moyen d'un $\mathrm{N}$ complété et déterminé un référent coprésent. Peu importe pour notre propos la nature réelle du référent (figuration? objet de la figuration ?). Ce qui vaut ici, c'est la fonction désignative de ces syntagmes.

On pourrait cependant légitimement s'interroger sur la pertinence de ces observations car, après tout, la désignation nominale a été et reste encore la forme standard de l'intitulation dans la peinture ${ }^{20}$. On verra donc dans la forme désignative des titres de Magritte le symptôme d'une conception spontanée du rapport du langage à la peinture, un rapport de désignation reliant les mots aux choses. Cette conception est tellement ancrée chez le peintre que lorsqu'il imagine pouvoir figurer des mots dans la toile elle-même, ce sont des séquences désignatives d'objets qui naissent alors sous le pinceau ${ }^{21}$. Cela est confirmé par un traitement spécifique de la composition qui aboutit, grâce à deux procédés distincts, à afficher ce rapport de désignation entre les mots et les choses.

Le premier procédé est celui de la division. Il concerne ces peintures « compartimentées » dans lesquelles une désignation est la contrepartie «naturelle » de la figuration, le pendant de l'image et/ou de l'idée. Ainsi la désignation peut occuper la place d'une image comme dans Le masque vide (1928) dans lequel toute une surface est divisée en quatre espaces, chacun d'eux accueillant en tant qu'objets peints, les désignations suivantes : ciel, corps bumain (ou forêt), rideau, façade de maison. Représentant un espace composé de quatre casiers dont l'un est occulté par un cache et dont les trois autres sont occupés respectivement par une main coupée, une pomme et un objet non identifié (pierre ou cire noires), Le musée d'une nuit de 1927 constitue une autre forme de dépiction par compartimentage. Mais les cellules sont ici occupées par des figurations d'objets. La division en cellules peut également accueillir des systèmes conjoints d'images et de mots. La Clef des songes de 1927, comme d'ailleurs celle de 1930, présentent plusieurs cases comportant chacune un dispositif d'étiquetage avec figuration d'objet dans la partie haute, légende désignative dans la partie basse ${ }^{22}$; dans la toile de 1927, un sac, un couteau suisse, une feuille d'arbre, une éponge comportent respectivement les légendes suivantes : Le ciel, L'oiseau, La table, L'éponge; dans celle de 1930, les couples image/légende sont les suivants: un œuf/l'Acacia, un soulier de femme/la Lune, un chapeau melon/la Neige, une bougie/le Plafond, un verre/l'Orange, un marteau/le Désert²3.

Un autre procédé aux effets analogues procède par réunion et non par division. Plusieurs toiles différentes peuvent être en effet associées pour composer un ensemble. Cette réunion constitue une seule et même œuvre dont la dépiction, éclatée,

${ }^{20}$ Cf F. Armengaud (1988) et B. Bosredon (1997).

21 A l'exception là encore du paradigme de Ceci n'est pas une pomme.

22 Sur la légende désignative, voir M.-S. Poli (1992) et B. Bosredon (1996).

${ }^{23}$ Selon Rémy Porquier, c'est le rapport entre l'image et le langage qu'on appréhende dans la lecture/observation et il faut comprendre que la relation entre le langage et l'image est ici tout à fait symétrique de sorte qu'on dire aussi bien que «Acacia a pour image un œuf » (communication personnelle).

Nous avons respecté les minuscules et les majuscules d'origine (J. Meuris, 1990); on voit que leur distribution n'est pas la même dans les deux toiles. 
se distribue en plusieurs toiles. On reconnaît là L'évidence éternelle de 1930, faite de cinq toiles disposées en colonne, qui figurent, ainsi présentées, les cinq parties d'un nu (le visage, une partie du buste et de la poitrine, le ventre et le sexe, les cuisses et les genoux, le bas des jambes et les pieds). Nous observons cependant que le procédé qui consiste à compartimenter la dépiction est certainement le plus répandu. Il livre toujours des solutions surprenantes, notamment cet arbre dont le tronc s'ouvre comme autant de boîtes recelant des objets inattendus: une maison éclairée, une boule blanche $e^{24}$.

En aménageant ainsi l'espace de la figuration, Magritte impose l'ordre de l'image au langage, celui du cadre et du tableau. Les désignations nominales, souvent les plus simples (un nom muni de son déterminant) et non la phrase ou le texte, que l'on voit courir parfois sur certains tableaux de Max Ernst ou de Miró, apparaissent dans ces conditions comme la structure linguistique la mieux adaptée à des places ménagées d'ordinaire pour des éléments de figuration. Si la confrontation image/langage est donc chez Magritte celle de la chose et de sa désignation, c'est qu'il y est conduit par la prééminence du cadre et de l'image dans l'établissement de cette relation. Il conçoit d'ailleurs la désignation que constitue un titre comme une sorte d'image verbale. «Le titre La durée poignardée, écrit-il, est lui-même une image (avec des mots) réunie à une image peinte ». Les images-mots entrent donc en relation avec des images peintes : «A propos des titres, il est à remarquer qu'ils doivent être des images qui s'unissent avec précision aux tableaux». Si Magritte peut s'interroger «naturellement» sur les relations entre l'image picturale et le langage, c'est qu'il conçoit l'une et l'autre dans l'ordre unique de la représentation.

\subsection{L'article défini}

La grande majorité des titres de Magritte commencent par un article défini. Le formulaire de référence, depuis ses premières toiles jusqu'à la fin de sa vie, est celui de la description définie : Le chant d'oisean (1914), Le jockey perdu (1926), La clef des champs (1933), L'échelle de feu (1939), La fée ignorante (1956), Le blanc-seing (1965) etc. Sur près de 140 titres répertoriés dans le René Magritte de Jacques Meuris, et si l'on écarte les trois titres phrases du type "Ceci n'est pas un X», on trouve plus de 120 titres en le/la/les pour une dizaine de titres commençant par un article zéro (Découverte de 1927) ou une préposition (Sans titre, 1926; En hommage à Mack Sennett, 1937) ${ }^{25}$. L'intitulation magrittienne est formellement classique. Elle répète fidèlement le formulaire traditionnel qui exprime la coïncidence de l'objet extrapictural et de sa figuration comme par exemple L'atelier de Le Nain ou de Vermeer, La charrette du boulanger de Michelin, La Bonne aventure de La Tour. Elle constitue également avec l'emploi du défini l'objet de la dépiction comme préexistant en quelque sorte à la dépiction même. Contrairement en effet à l'article zéro qui est la marque d'un opérateur indexical de "présentification ${ }^{26}$ dans le site d'étiquetage constitué par la coprésence de la

\footnotetext{
${ }^{24}$ Il s'agit de deux toiles de 1948 ayant le même titre La voix du sang.

${ }^{25}$ Ces titres sont des sortes de «métatitres » et ne peuvent être rangés dans la même catégorie que les autres.

${ }^{26}$ B. Bosredon (1997, p. 63$)$
} 
dépiction et du titre, l'article LE (le/la/les) décroche la détermination du site. L'article défini dans L'échelle du feu constitue donc la définitude indépendamment de la coprésence de la dépiction, quelle que soit par ailleurs la nature de cette dépiction. Le système $ø /($ le/la/les) correspond par conséquent à deux valeurs différentes de la définitude. D'un côté, $\varnothing$ marque la définitude d'une définitude indexicale in situ, de l'autre LE marque la construction d'une définitude en dehors de tout site d'étiquetage, c'est-à-dire une définitude en discours ${ }^{27}$.

C'est donc dans les limites mêmes de l'intitulation classique que Magritte s'interroge sur le rapport entre les images et les mots. C'est la raison pour laquelle ses titres sont des énigmes. Ils stipulent en effet ce qui est visiblement non adéquat : les figurations ne correspondent pas à ce qui les désignent. Il n'y a pas vraiment dénégation de la double équation classique $:$ " peinture $=$ image de chose $=$ la chose même garantie par le titre ». C'est pourquoi l'observateur-lecteur prend au sérieux la relation entre le titre et la dépiction et cherche le «vrai sens » de l'image, estimant (à juste titre...) qu'une raison suffisante doit bien valider ces liens étranges. D'autre part, Magritte reste également dans les limites discursives traditionnelles en constituant avec l'article défini une entité unique qui préexiste à la peinture. Entité cachée certes, entité à libérer des chaines de nos habitudes de voir ou de pensée, mais entité unique cependant, assez proches par la manière de les désigner en tout cas de ce qui constitue une ontologie. L'intitulation de Magritte est classique en ce qu'elle nomme des objets. Elle est surréaliste en ce qu'elle nomme des objets «autres». Les quelques titresphrases du type «Ceci n'est pas un $\mathrm{X} »^{28}$ ne font pas sortir Magritte de ce cadre. Bien au contraire. Si ces titres ne sont pas en effet des désignations, ils interrogent néanmoins toujours sur la pertinence du titre désignatif. Ils disent explicitement à la fois qu'un titre peut être trompeur mais qu'il reste une désignation d'objet. Ces titres ne constituent des formes de «méta-intitulation » que parce qu'on les mesure à l'aune de l'intitulation désignative. Mais le plus surprenant c'est que l'objet métadiscursif de ce formulaire inédit reste la désignation.

Il est possible que Magritte n'ait pu échapper à cette emprise pour d'autres raisons encore. Il en est une que nous mentionnerons tant elle reste active et encore omniprésente dans les pratiques contemporaines de l'intitulation. Il s'agit du statut de nom propre donné aux titres de peintures. C'est une dimension du titre toujours présente, encore aujourd'hui. Il est certain que cette fonction de nomination propre fraye la voie, plus que tout autre, à la désignation nominale munie d'une détermination définie.

27 ibid. p. 68.

${ }^{28}$ Cette formule a même servi pour intituler un mixte de peinture et d'objet; il s'agit d'une toile représentant une tranche de brie ou de camembert sous une cloche intitulée Ceci est un morceau de fromage. Le dispositif qui est un objet en trois dimensions conduit le peintre à inverser le sens de la dénégation. 
Les titres de Magritte : surprise et convenance discursive

\section{Conclusion}

Magritte appartient à cette famille de peintres pour lesquels l'intitulation est chose nécessaire. Titre et dépiction collaborent simultanément à l'unité d'une œuvre, le langage et l'image concourant ensemble à sa production. Cette unité est chez Magritte celle d'un rapport énigmatique qui abolit le vieux rapport traditionnel du titre à la peinture en faisant de celui-ci l'interprétant de celle-là, sous la forme de la désignation ou du commentaire. Le titre constitue alors, avec la peinture, une « surprise ». On a pu cependant observer au cours de notre parcours que cette surprise se manifestait dans la forme d'une intitulation conforme paradoxalement à la signalétique de la nomination de la peinture classique où dominent les désignations nominales définies. C'est certainement l'expression de l'inattendu dans un formulaire convenu qui conduit à la tonalité énigmatique des titres de Magritte.

Bernard BOSREDON

14 rue Ulysse Benne

94700 Maisons-Alfort

elisabos@club-internet.fr bosredon@univ-paris3.fr 


\section{BIBLIOGRAPHIE}

ARMENGAUD Françoise, 1988, Titres, Paris, Klincksieck.

BOSREDON Bernard, 1996, "Titre et légende: absence de marque et marque d'absence», Absence de marques et représentation de l'absence, Rennes, les PUR, pp. 349-367.

BOSREDON Bernard, 1997, Les titres de tableaux, une pragmatique de l'identification, Paris, P.U.F.

FOUCAULT Michel, 1973, Ceci n'est pas une pipe, Paris, Fata Morgana.

HOFSTADTER Douglas, 1985, Gödel, Escher, Bach. Les brins d'une guirlande éternelle, Paris, InterEditions, pp. 789-796.

PÉREC Georges, 1999, Un cabinet d'amateur, Paris, Librairie Générale Française, Livre de Poche.

MAGRITTE René, 1979, Ecrits complets, France, Flammarion.

MAGRITTE René, 1990, Lettres à André Bosmans, France, Seghers Isy Brachot.

MEURIS Jacques, 1990, René Magritte, Cologne, Taschen.

NOUGE Paul, 1943, René Magritte ou Les images défendues, Bruxelles, Auteurs associés.

POLI Marie-Sylvie, 1992, «Le parti pris des mots dans l'étiquette : une approche linguistique », Publics et musées, 1, PUL. 